\title{
Whole-Brain Mapping of Direct Inputs to Dopamine D1 and D2 Receptor-Expressing Medium Spiny Neurons in the Posterior Dorsomedial Striatum
}

\author{
Jiayi Lu, Yifeng Cheng, Xueyi Xie, Kayla Woodson, Jordan Bonifacio, Emily Disney, Britton Barbee, \\ Xuehua Wang, Mariam Zaidi, and (DJun Wang
}

https://doi.org/10.1523/ENEURO.0348-20.2020

Department of Neuroscience and Experimental Therapeutics, College of Medicine, Texas A\&M University Health Science Center, Bryan, TX 77807

\begin{abstract}
The posterior dorsomedial striatum (pDMS) is mainly composed of medium spiny neurons (MSNs) expressing either dopamine D1 receptors (D1Rs) or D2Rs. Activation of these two MSN types produces opposing effects on addictive behaviors. However, it remains unclear whether pDMS D1-MSNs or D2-MSNs receive afferent inputs from different brain regions or whether the extrastriatal afferents express distinct dopamine receptors. To assess whether these afferents also contained D1Rs or D2Rs, we generated double transgenic mice, in which D1R-expressing and D2R-expressing neurons were fluorescently labeled. We used rabies virus-mediated retrograde tracing in these mice to perform whole-brain mapping of direct inputs to D1-MSNs or D2-MSNs in the pDMS. We found that D1-MSNs preferentially received inputs from the secondary motor, secondary visual, and cingulate cortices, whereas D2-MSNs received inputs from the primary motor and primary sensory cortices, and the thalamus. We also discovered that the bed nucleus of the stria terminalis (BNST) and the central nucleus of the amygdala (CeA) contained abundant D2Rexpressing, but few D1R-expressing, neurons in a triple transgenic mouse model. Remarkably, although limited D1R or D2R expression was observed in extrastriatal neurons that projected to D1-MSNs or D2-MSNs, we found that cortical structures preferentially contained D1R-expressing neurons that projected to D1-MSNs or D2-MSNs, while the thalamus, substantia nigra pars compacta (SNc), and BNST had more D2R-expressing cells that projected to D2-MSNs. Taken together, these findings provide a foundation for future understanding of the pDMS circuit and its role in action selection and reward-based behaviors.
\end{abstract}

Key words: BNST; CeA; dopamine D1 and D2 receptors; posterior dorsomedial striatum; rabies virus-mediated retrograde monosynaptic tracing; whole-brain mapping

\section{Significance Statement}

The dorsomedial striatum (DMS) is a brain region that has critical roles in drug addiction. The DMS receives and integrates information from multiple other regions, such as the cortex and thalamus. These extrastriatal inputs onto two DMS cell types, D1-neurons and D2-neurons, provide a substrate for how the brain segregates information process, as D1-neurons positively and D2-neurons negatively control addiction-associated behaviors. The present study found that the cortex and other brain regions that are involved in motivational and decision-making behaviors preferentially targeted D1-neurons, whereas the thalamus, which plays essential roles in behavioral flexibility, preferentially projected to D2-neurons. These findings of distinct innervations of D1-neurons and D2-neurons provide a foundation for future studies of DMS function.

Received August 12, 2020; accepted December 19, 2020; First published December 30, 2020.

The authors declare no competing financial interests.
Author contributions: J.L., Y.C., and J.W. designed research; J.L., Y.C., X.X., B.B., and X.W. performed research; J.L., Y.C., X.X., K.W., J.B., E.D., B.B., M.Z., and J.W. analyzed data; J.L., Y.C., and J.W. wrote the paper. 


\section{Introduction}

The basal ganglia has critical roles in movement control and action selection (Balleine et al., 2009). The striatum, which provides the primary input to the basal ganglia, receives and integrates information from cortical, thalamic, and limbic structures before passing this to the basal ganglia for an appropriate action (Bamford et al., 2018). Growing evidence indicates that the dorsomedial striatum (DMS) is involved in the control of goal-directed actions and drug-seeking behaviors (Volkow et al., 2006; Wang et al., 2010; Ma et al., 2018). Two distinct subtypes of medium spiny neuron (MSN) make up as much as $95 \%$ of neurons within the DMS (Gerfen and Surmeier, 2011; Cheng et al., 2017). One type of MSN expresses dopamine D1 receptors (D1Rs) and projects to the substantia nigra pars reticulata (SNr), forming the direct pathway (Kreitzer and Malenka, 2008; Gerfen and Surmeier, 2011). The other type of MSN expresses dopamine D2 receptors (D2Rs) and projects to the external globus pallidus $(\mathrm{GPe})$, forming the indirect pathway (Kreitzer and Malenka, 2008; Gerfen and Surmeier, 2011). Activation of D1R-expressing MSNs (D1-MSNs) or D2R-expressing MSNs (D2-MSNs) produces opposing effects on movement and reward-related behaviors (Gerfen et al., 2013; Cheng et al., 2017; Lüscher et al., 2020). Thus, many learned responses require the coordinated activation of the direct pathway and inhibition of the indirect pathway in the DMS. Importantly, recent studies have found that the posterior region of the DMS (pDMS) is likely to play a greater role in goal-directed behavior than the anterior DMS (aDMS; Yin and Knowlton, 2004; Yin et al., 2005b; Regier et al., 2015). The pDMS is necessary for the integration of action-outcome associations and expression of goal-directed learning (Yin and Knowlton, 2004; Yin et al., 2005a,b; Corbit and Janak, 2010; Shiflett et al., 2010). Additionally, a recent anatomic study also found different cortical inputs to the aDMS and pDMS (Hunnicutt et al., 2016). While the aDMS mainly receives inputs from the orbital, prelimbic, infralimbic, and anterior cingulate cortices, the pDMS primarily receives inputs from the motor, orbital, cingulate, and visual cortices (Hunnicutt et al., 2016). While the innervation of D1-MSNs and D2-MSNs in the dorsal striatum has been studied using the rabies virus-mediated retrograde monosynaptic tracing system (Wall et al., 2013), it remains unclear whether D1-MSNs and D2-MSNs in the pDMS receive distinct inputs from other brain areas.

In addition to receiving excitatory glutamatergic inputs from the cortex, thalamus, and amygdala, DMS D1-MSNs and D2-MSNs are modulated by dopaminergic inputs from the substantia nigra pars compacta (SNc; Bamford et al., 2018). Dopamine can alter MSN activity by acting

\footnotetext{
This work was supported by National Institute on Alcohol Abuse and Alcoholism Grants R01AA021505, R01AA027768, and U01AA025932 (to J.W.).

Correspondence should be addressed to Jun Wang at jwang188@tamu.edu.

https://doi.org/10.1523/ENEURO.0348-20.2020

Copyright (C) 2021 Lu et al.

This is an open-access article distributed under the terms of the Creative Commons Attribution 4.0 International license, which permits unrestricted use, distribution and reproduction in any medium provided that the original work is properly attributed.
}

on their postsynaptic D1Rs or D2Rs (Gerfen and Surmeier, 2011; Cheng et al., 2017; Lüscher et al., 2020) or by acting on presynaptic receptors (Wang and Pickel, 2002; Dumartin et al., 2007; Lu et al., 2019). Electron microscopy studies have provided evidence that cortical fibers in the dorsal striatum contain both D1Rs and D2Rs (Wang and Pickel, 2002; Dumartin et al., 2007), although D1R immunoreactivity was observed less frequently (Dumartin et al., 2007). A recent study also showed that cortical neurons that projected to the DMS preferentially expressed D2Rs, rather than D1Rs (Lu et al., 2019). However, these studies could not determine which specific DMS neuronal types received the D1R-expressing or D2R-expressing inputs.

In this study, we assessed D1R and D2R expression patterns in extrastriatal neurons by generating cell-typespecific Cre-expressing double transgenic mice, in which D1R-expressing and D2R-expressing cells were labeled by a fluorescent protein. To map direct inputs to D1MSNs or D2-MSNs, we used the rabies virus-mediated retrograde tracing approach; this allowed us to label neurons that projected monosynaptically to D1-MSNs or D2MSNs (Wall et al., 2013; Ogawa and Watabe-Uchida, 2018). We discovered that neurons in the orbital frontal, secondary motor, visual, and cingulate cortices preferentially targeted pDMS D1-MSNs. In contrast, neurons in the thalamus, primary motor cortex, and primary sensory cortex preferentially projected to pDMS D2-MSNs. Furthermore, our triple transgenic mouse models showed that the bed nucleus of the stria terminalis (BNST) and the central nucleus of the amygdala (CeA) contained abundant D2R-expressing, but few D1R-expressing, neurons. Lastly, we found that while the number of D1R-expressing or D2R-expressing neurons that projected to pDMS MSNs was low, they exhibited distinct distribution across different brain regions. These findings lay a foundation for an improved understanding of how the pDMS organizes information from multiple upstream brain regions to determine an action.

\section{Materials and Methods}

\section{Reagents}

Two Cre-dependent (Flex) adeno-associated virus (AAV) serotype eight vectors were employed in this study; one expressed rabies glycoprotein (RG; AAV8-Flex$R G)$ and the other expressed an avian membrane EnvA receptor protein (TVA) and mCherry (AV8-Flex-TVA$m$ Cherry). These helper viruses were purchased from the University of North Carolina Vector Core. The pseudotyped rabies viruses, EnvA-SAD $\Delta$ G-mCherry and EnvA-SAD $\Delta$ G-GFP $\left(2.04 \times 10^{8} \mathrm{TU} / \mathrm{ml}\right)$, were obtained from the Salk Institute Vector Core. All other reagents were purchased from Sigma.

\section{Animals}

Drd1a-Cre (D1-Cre) and Drd2-Cre (D2-Cre) mice were acquired from the Mutant Mouse Regional Resource Center. Snap25 mice and D1-tdTomato mice were purchased from The Jackson Laboratory. D1-Cre or D2Cre mice were crossed with Snap25 mice on a C57BL/ 6J background to produce D1-Cre;Snap25 or D2-Cre; 
Snap25 offspring. D2-Cre mice were also crossed with D1-tdTomato on a C57BL/6J background to produce D1-tdTomato;D2-Cre offspring. Mouse genotypes were determined by PCR analysis of Cre or the fluorescent protein gene in tail DNA (Cre for D1-Cre and D2-Cre mice; tdT for D1-tdTomato; GFP for Snap25 mice; Wang et al., 2011, 2015; Cheng et al., 2017, 2018; Ma et al., 2018; Wei et al., 2018; Lu et al., 2019). Mice were group-housed at $23^{\circ} \mathrm{C}$ with a $12 / 12 \mathrm{~h}$ light/dark cycle (lights on at 11 P.M.). Food and water were provided ad libitum. Male two- to three-month-old mice were used in this study. All animal care procedures and experimental protocols were approved by the Institutional Animal Care and Use Committee and were performed in agreement with the National Research Council Guide for the Care and Use of Laboratory Animals.

\section{Stereotaxic virus infusion}

Stereotaxic viral infusions were performed as described previously (Wang et al., 2015; Cheng et al., 2017; Huang et al., 2017; Ma et al., 2017, 2018; Lu et al., 2019; Roltsch Hellard et al., 2019). Briefly, mice were anesthetized using isoflurane and mounted in a rodent stereotaxic frame (Kopf). The skin was opened to uncover the skull and expose bregma, lamda, and the location of the desired injection site. A three-axis micromanipulator was used to measure the spatial coordinates for bregma and lamda. Small drill holes were made in the skull at the appropriate coordinates, according to the Paxinos atlas (Franklin and Paxinos, 2007). Two microinjectors were loaded with $0.5 \mu \mathrm{L}$ of a $1: 1$ mixture of AAV8-Flex-RG and AAV8-FlexTVA-mCherry and then lowered into the pDMS (AP: 0.0 $\mathrm{mm}, \mathrm{ML}: \pm 1.87 \mathrm{~mm}, \mathrm{DV}:-2.90 \mathrm{~mm})$. This helper virus mixture was infused into the brain at a rate of $0.1 \mu \mathrm{l} / \mathrm{min}$. To avoid the backflow of the virus, microinjectors were left in place for $10 \mathrm{~min}$ after the infusion was complete and were then removed. The skin was sutured, and the mice were allowed to recover for three weeks before the infusion of pseudotyped rabies virus (EnvA-SAD $\Delta$ G-mCherry or EnvA-SAD $\Delta$ G-eGFP). The rabies virus was injected at the same site and using the same injection volume as the initial helper virus injection. To prevent coincident rabies infection along the AAV injection tract, the rabies virus was infused at an angle of $10^{\circ}$ (Wall et al., 2013) into adapted coordinates (AP, $0.0 \mathrm{~mm}$; ML, $\pm 2.42 \mathrm{~mm}$; DV, $-2.94 \mathrm{~mm}$ ). The modified coordinates were calculated by measuring from the midline and parallel to the dorsal-ventral axis.

\section{Confocal imaging and cell counting}

Rabies virus was allowed to replicate and spread for $7 d$ before perfusing the mice intracardially with $4 \%$ paraformaldehyde (PFA) in PBS (Wall et al., 2013). The brains were extracted and postfixed overnight in 4\% PFA/PBS solution, followed by dehydration in $30 \%$ sucrose. Each whole brain was sectioned serially into $50-\mu \mathrm{m}$ coronal sections using a cryostat. We mounted sections in a one in four series. Confocal images were obtained using a confocal laser-scanning microscope (Fluoview 1200,
Olympus). Fluorescent images were reconstructed in three dimensions, and cell counts from these scans were manually acquired using the Bitplane Imaris 8.3.1 (Bitplane), as previously reported (Wei et al., 2018; Lu et al., 2019). Green and red neurons were counted using the Spot module within Imaris, which also calculated colocalization. Brain structures were registered using the Paxinos mouse atlas as a reference (Franklin and Paxinos, 2007).

\section{Statistical analysis}

Data were analyzed by two-tailed $t$ test, two-way ANOVA with repeated measurement (RM), or one-way ANOVA followed by the Tukey's post hoc test. Significance was determined if $p<0.05$. Statistical analysis was conducted by SigmaPlot.

\section{Results}

\section{Identification of distinct whole-brain extrastriatal inputs to D1-MSNs and D2-MSNs in the pDMS}

To compare afferent inputs onto pDMS D1-MSNs and D2-MSNs, we employed rabies-mediated monosynaptic retrograde tracing in D1-Cre;Snap25 and D2-Cre;Snap25 mice, in which D1-MSNs or D2-MSNs selectively expressed Cre (and thus GFP), respectively (Madisen et al., 2015; Wei et al., 2018; Lu et al., 2019). In these mouse lines, the pDMS was injected with Cre-dependent AAVs that expressed TVA-mCherry and RG (Fig. 1A). TVA facilitated neuronal infection by the pseudotyped rabies virus, while RG facilitated retrograde monosynaptic spread of the rabies virus from infected neurons (Wall et al., 2013; Ogawa and Watabe-Uchida, 2018). Three weeks after the infusion of these vectors, we injected the glycoprotein-deleted pseudotyped rabies virus, EnvA-SAD $\Delta$ G-mCherry, at the same pDMS location using an angled injection tract (Fig. 1B) to prevent coincident infection (Wall et al., 2013). This strategy of selectively expressing TVA and RG in Cre-expressing D1-MSNs in D1-Cre;Snap25 mice or D2MSNs in the D2-Cre;Snap25 mice meant that the rabies virus selectively infected these neurons and spread to neurons with monosynaptic inputs to them (Fig. 1C). One week after the rabies virus infusions, serial coronal $50-\mu \mathrm{m}$ sections of the whole brain were prepared, and every fourth section was imaged using confocal laser-scanning microscopy. We observed intense GFP expression in the striatum and at D1-MSN projection targets, including the GPe (Fig. 1Div), entopeduncular nucleus (Fig. 1Dv), and SNr (Fig. 1Dvii), and at D2-MSN projection targets such as the GPe (Fig. 1Dxi). We also observed a large number of mCherry-positive extrastriatal neurons with monosynaptic connections to D1-MSNs (Fig. 1Di-Dvii) or D2MSNs (Fig. 1Dviii-Dxiv); these were located in the cortex (Fig. 1Di-Dxiv), BNST (Fig. 1Diii, Dx), GPe (Fig. 1Div, Dxi), amygdala (Fig. 1Dv, Dxii), thalamus (Fig. 1Dvi, Dxiii), and the midbrain (Fig. 1Dvii, Dxiv).

These rabies-labeled afferent (mCherry-positive) neurons in both hemispheres were counted relative to brain region boundaries. The total number of extrastriatal mCherry-positive neurons did not differ significantly 


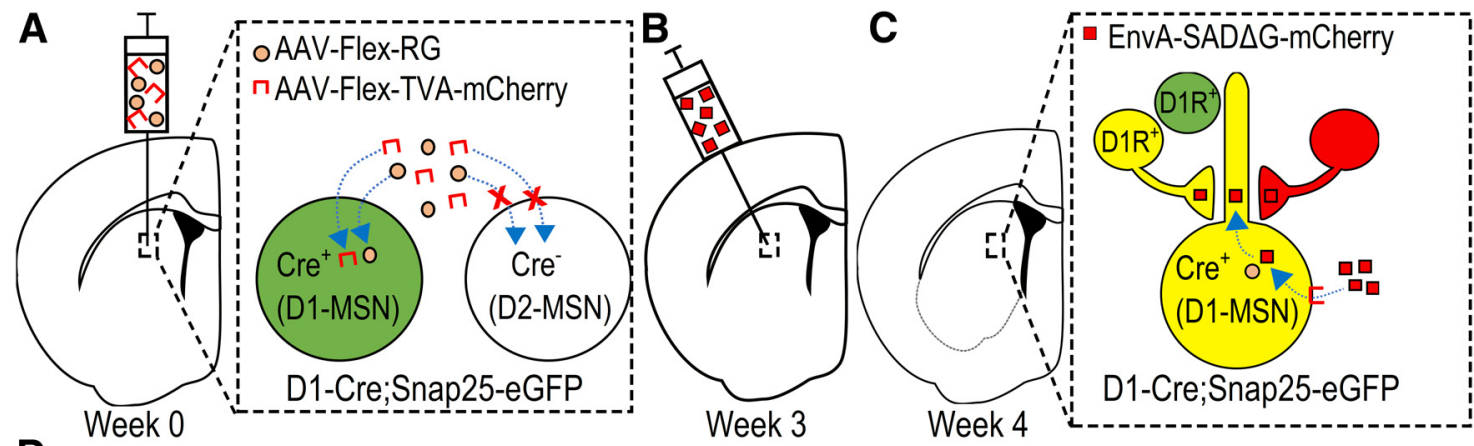

D

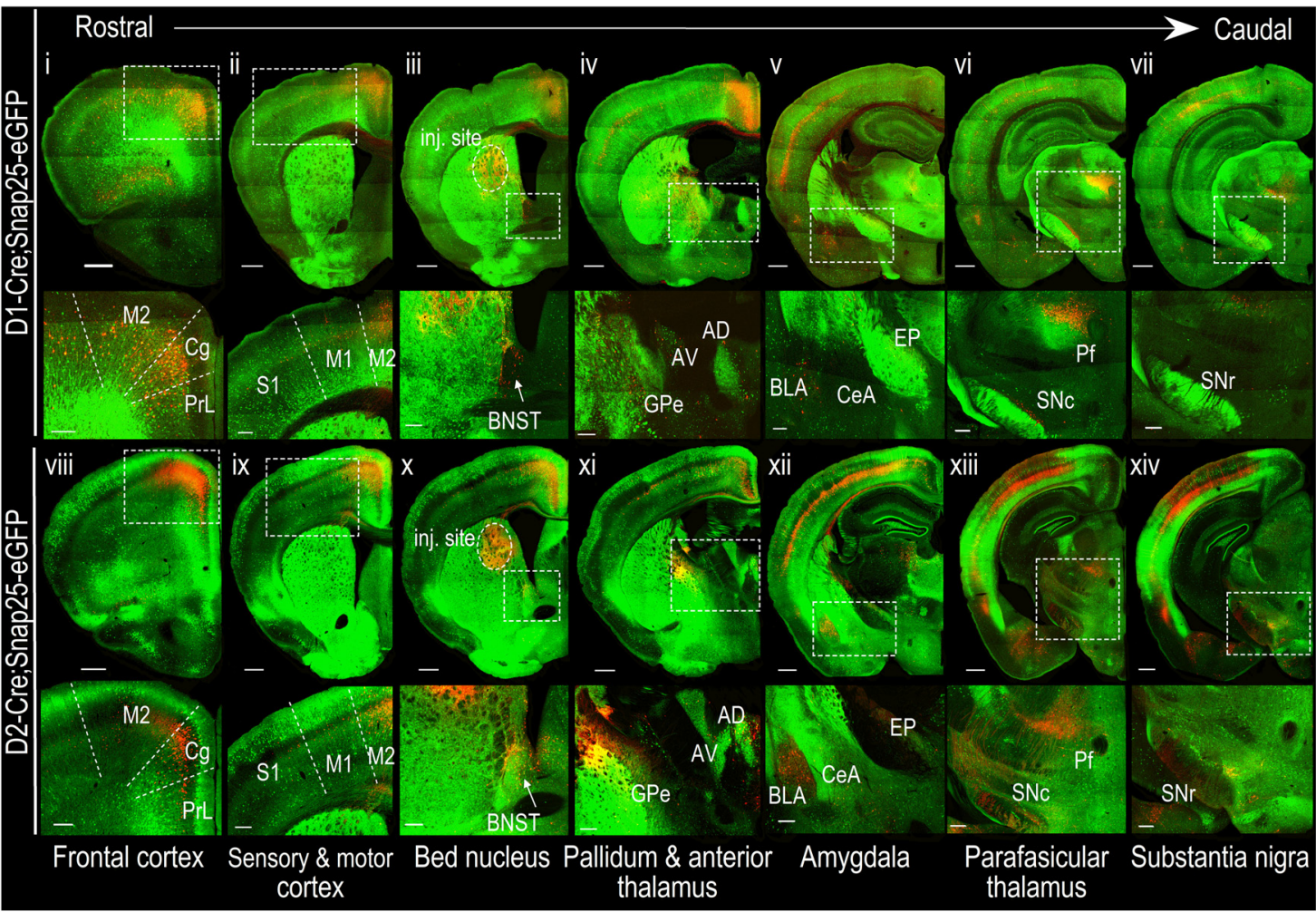

$\mathbf{E}$

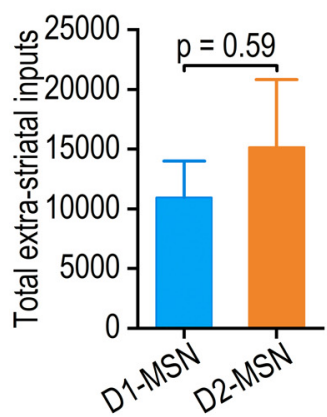

$\mathbf{F}$

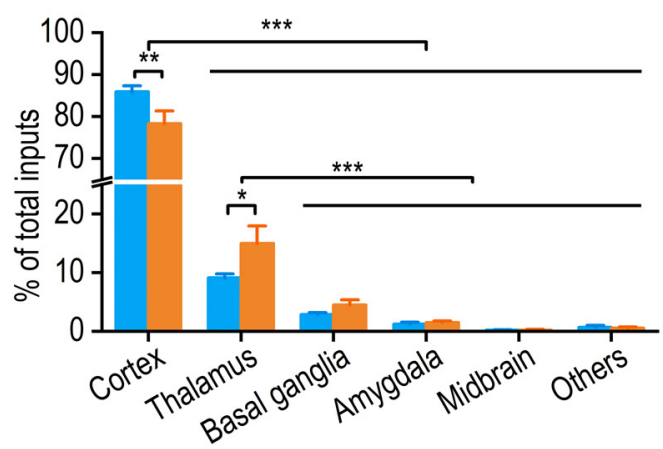

Figure 1. Rabies virus-mediated retrograde monosynaptic whole-brain tracing of neurons projecting to D1-MSNs and D2-MSNs in the pDMS. $\boldsymbol{A}, \boldsymbol{B}$, The experimental design used to trace neurons with afferent inputs to D1-MSNs in the pDMS. The design shows how we employed D1-Cre;Snap25 transgenic mice, in which D1-MSNs selectively expressed Cre and GFP (green). Note that the same approach was repeated in D2-Cre;Snap25 and D1-tdTomato;D2Cre mice, where we traced afferent inputs to D2-MSNs. Selective rabies infection of D1-MSNs was achieved by injecting the pDMS of these mice with Cre-dependent helper viruses expressing an avian membrane EnvA receptor protein (TVA) and RG (AAV-Flex-TVA-mCherry and AAV-Flex-RG) three weeks before injection of a modified rabies virus (EnvA-SAD $\triangle$ G-mCherry) into the same area at a $10^{\circ}$ angle. $\boldsymbol{C}$, One week after rabies injection, the rabies virus had specifically infected TVA-expressing D1-MSNs and spread retrogradely from RG-expressing D1-MSNs to neurons with monosynaptic connections with them. Note that extrastriatal neurons with monosynaptic connections to RG-expressing 
continued

D1-MSNs expressed rabies-derived mCherry (red). mCherry-expressing neurons that also contained D1Rs and thus expressed Credriven GFP (green) appeared yellow. Extrastriatal neurons that expressed D1Rs but did not make any connections with RG-expressing D1-MSNs were labeled green. $\boldsymbol{D}$, Representative confocal images of rabies virus-labeled mCherry-expressing neurons (red) that project to D1-MSNs (i-vii) or D2-MSNs (viii-xiv) throughout the brain in the D1-Cre;Snap25 or D2-Cre;Snap25 mice, respectively. Rows 2 and 4 show enlarged images of the boxed areas in rows 1 and 3, respectively. Note that there were extensive mCherry-positive neurons in the cortex (i-xiv), BNST (iii, x), GPe (iv, xi), amygdala (v, xii), thalamus (vi, xiii), and midbrain (vii, xiv). M2, secondary motor cortex; Cg, cingulate cortex; PrL, prelimbic cortex; S1, primary sensory cortex; M1, primary motor cortex; BNST, bed nucleus of the stria terminalis; GPe, globus pallidus external; $\mathrm{AD}$, anterior dorsal thalamus; $\mathrm{AV}$, anterior ventral thalamus; BLA, basolateral amygdala; CeA, central amygdala; EP, entopeduncular nucleus; Pf, parafascicular thalamic nucleus; SNc, substantia nigra pars compacta; SNr, substantia nigra pars reticulata; inj. site, injection site. Scale bars: $500 \mu \mathrm{m}$ (rows 1 and 3 ) and $200 \mu \mathrm{m}$ (rows 2 and 4). $\boldsymbol{E}$, There was no significant difference between the total number of extrastriatal neurons with projections to D1-MSNs or D2MSNs; unpaired $t$ test. $\boldsymbol{F}$, Extrastriatal inputs onto D1-MSNs (blue) versus D2-MSNs (orange); ${ }^{*} p<0.05,{ }^{* *} p<0.01,{ }^{* * *} p<0.001$; two-way RM ANOVA followed by Tukey's test for the indicated comparisons; $n=5$ mice for D1-MSNs (D1-Cre;Snap25 mice) and $n=8$ mice for D2-MSNs (4 D2-Cre;Snap25 mice and 4 D1-tdTomato;D2-Cre mice; $\boldsymbol{E}, \boldsymbol{F}$ ).

between D1-Cre;Snap25 and D2-Cre;Snap25 mice $\left(t_{(11)}=-0.55, p>0.05\right.$; Fig. $\left.1 E\right)$. Since both AAV8-FlexTVA-mCherry and the rabies virus expressed mCherry, this did not allow for selective visualization of starter cells, which were infected by both the rabies virus and AAV8-Flex-RG. Thus, labeled neuron counts from any given brain region were normalized to the total inputs to the pDMS detected within each animal. This approach was used to account for any inter-animal differences in viral infusion. This analysis identified significant differences in the extent of inputs from distinct brain regions $\left(F_{(5,55)}=627.19, p<0.001\right.$; Fig. $\left.1 F\right)$. Specifically, the majority of inputs onto pDMS D1-MSNs and D2-MSNs arose from the cortex $(p<0.001$ for cortex vs thalamus, basal ganglia, amygdala, midbrain, and others; Fig. 1F), and from the thalamus $(p<0.001$ for thalamus vs basal ganglia, amygdala, midbrain, and others; Fig. $1 F$ ). Interestingly, cortical neurons preferentially innervated D1-MSNs versus D2-MSNs, whereas thalamic neurons preferentially connected with D2-MSNs versus D1-MSNs (cortical neurons: $p<0.01$; thalamic neurons: $p<0.05$; Fig. $1 F$ ).

Both the cortex and thalamus contain many subregions. Within the cortex, the most prominent inputs to D1-MSNs (expressed as a percentage of total inputs) were from the orbital frontal cortex $(6.62 \pm 1.45 \%)$, primary $(6.17 \pm 1.31 \%)$ and secondary $(21.93 \pm 1.47 \%)$ motor cortices, primary sensory cortex $(10.53 \pm 1.25 \%)$, and cingulate cortex (18.59 $\pm 1.27 \%$; Fig. 2). For D2MSNs, the equivalent percentages were $5.20 \pm 0.93 \%$, $8.40 \pm 0.94 \%, 17.13 \pm 0.42 \%, 15.31 \pm 1.52 \%$, and $11.66 \pm$ $1.10 \%$ of total D2-MSN inputs, respectively (Fig. 2). Within the thalamus, the parafascicular nucleus, mediodorsal thalamic nucleus, and central thalamic nucleus gave rise to the majority of thalamostriatal inputs to D1-MSNs and D2MSNs $(2.75 \pm 0.77 \%, 2.21 \pm 0.40 \%$, and $1.28 \pm 0.21 \%$ of total D1-MSN inputs, respectively; and $2.97 \pm 1.10 \%$, $2.29 \pm 0.31 \%$, and $3.10 \pm 0.86 \%$ of total D2-MSN inputs, respectively; Fig. 2). These data suggest that pDMS MSNs receive inputs primarily from the cortex and thalamus, which preferentially project to D1-MSNs and D2MSNs, respectively.

Previous studies have demonstrated that the striatum plays a critical role in sorting sensory, motor, and reward information arriving from two major excitatory sources: the cortex and thalamus (Lovinger, 2010; Bamford et al.,
2018). Differential activation of the direct and indirect pathways subsequently results in the selection and execution of appropriate responses. Therefore, we next analyzed whether there were cortical or thalamic subregions that preferentially targeted D1-MSNs or D2-MSNs. Although many cortical structures provided similar proportions of the inputs to D1-MSNs and D2-MSNs, we identified some cortical regions that showed a considerable bias in their synaptic input to a specific cell type. The secondary motor, cingulate, and secondary visual cortices provided significantly higher proportions of the inputs to D1-MSNs, as compared with D2-MSNs $(p<0.001$, 0.001 , and 0.05 , respectively, Figs. $1 D, 2)$. This trend was also observed in the orbital frontal cortex $(p=0.055$; Fig. 2). In contrast, the primary motor and primary sensory cortices provided more inputs to D2-MSNs than to D1MSNs $(p<0.05$ and 0.001 , respectively; Figs. $1 D, 2)$. The other major source of glutamatergic input to the striatum, the thalamostriatal projections, also showed some biased connectivity. Both the central and ventromedial thalamic nuclei provided significantly higher proportions of inputs to D2-MSNs than to D1-MSNs $(p<0.05$; Figs. $1 D, 2)$. In addition to these cortical and thalamic inputs, the amygdala provides glutamatergic inputs to the DMS. The present study found similar proportions of synaptic inputs to D1-MSNs and D2-MSNs from the central, basal, and lateral amygdala $(p>0.05$ for all areas; Figs. $1 D, 2)$.

We also compared the projections from the SNc, a known dopaminergic input to the DMS, to D1-MSNs and D2-MSNs (Surmeier et al., 2007). Consistent with a previous report (Wall et al., 2013), we found that a relatively small proportion of the total inputs were from the $\mathrm{SNc}$, and there was no significant difference between the percentages of these inputs to D1-MSNs and D2MSNs ( $p>0.05$; Figs. $1 D, 2)$. Lastly, we compared the GABAergic inputs from the GPe to the DMS (Lee et al., 2004; Hernández et al., 2015) and found significantly greater D2-MSN innervation ( $p<0.05$; Figs. $1 D, 2)$.

Taken together, these observations demonstrated input specificity onto two MSN subtypes in the pDMS. We discovered that the secondary motor, secondary visual, and cingulate cortices preferentially targeted D1-MSNs, whereas inputs from the thalamus, primary motor cortex, and primary sensory cortex preferentially projected to D2-MSNs. Consequently, the cortex and 


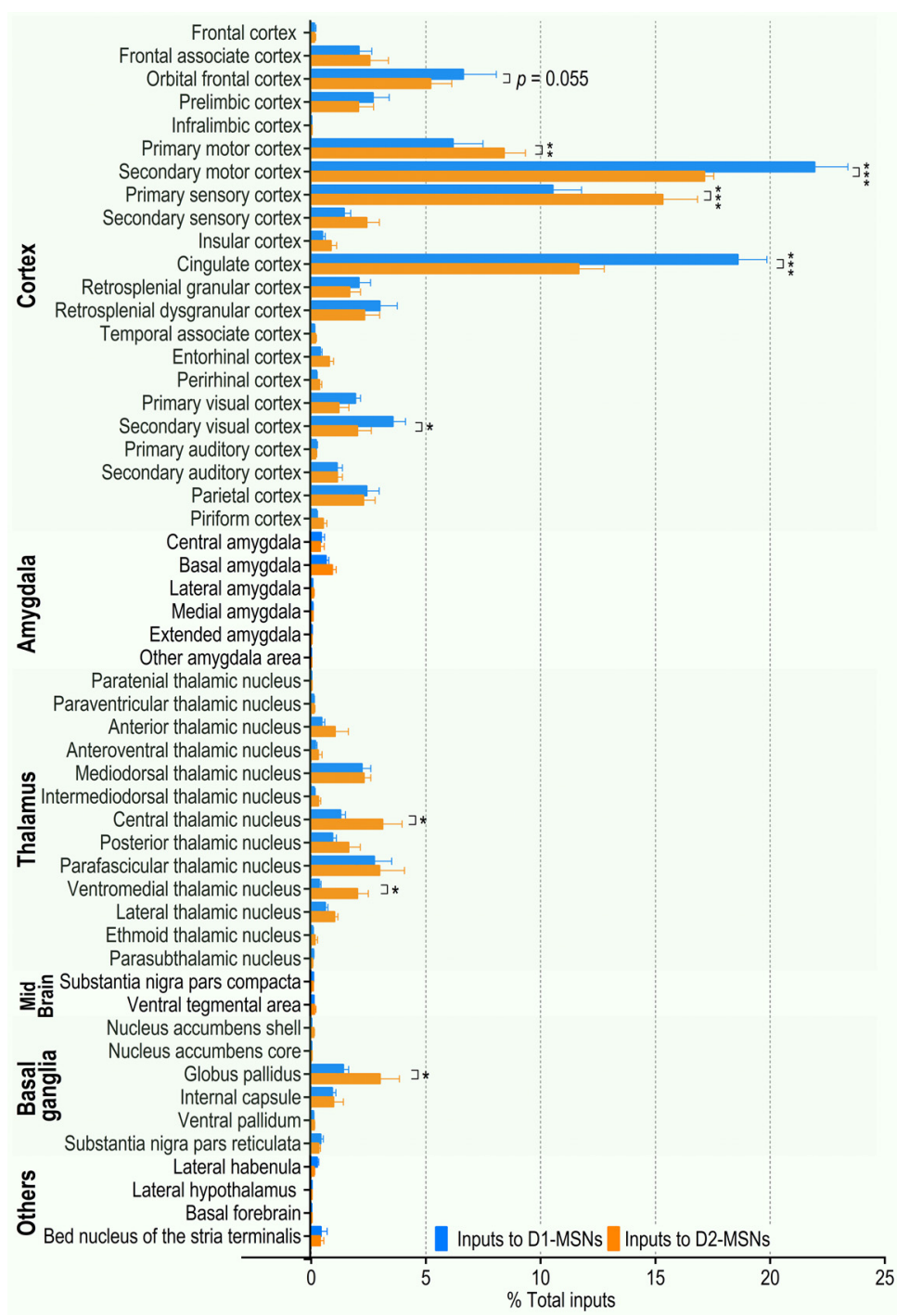

Figure 2. Summary of brain-wide monosynaptic inputs to pDMS D1-MSNs and D2-MSNs. The majority of direct synaptic inputs to the pDMS arose in the cortex and thalamus. Analysis of the normalized distribution of rabies virus-labeled neurons showed that the orbital frontal cortex, secondary motor cortex, cingulate cortex, and secondary visual cortex preferentially projected to D1-MSNs versus D2-MSNs. The primary motor cortex, primary sensory cortex, central thalamic nucleus, ventromedial thalamic nucleus, and globus pallidus preferentially innervated D2-MSNs versus D1-MSNs. The extrastriatal inputs onto D1-MSNs (blue) or D2-MSNs (orange) are expressed as a percentage of the total inputs to these cell types; ${ }^{*} p<0.05,{ }^{* *} p<0.01$, ${ }^{* * *} p<0.001$; two-way RM ANOVA followed by Tukey's test; $n=5$ mice for D1-MSNs (D1-Cre;Snap25 mice) and $n=8$ mice for D2-MSNs (4 D2-Cre;Snap25 mice and 4 D1-tdTomato;D2-Cre mice).

thalamus preferentially projected to pDMS D1-MSNs and D2-MSNs, respectively.

\section{Preferential expression of D2Rs versus D1Rs in the BNST and CeA}

Having identified the distinct extrastriatal inputs to D1MSNs versus D2-MSNs, we next asked whether there are any differences in the presynaptic inputs onto D1-MSNs or D2-MSNs in terms of their D1R and D2R expression, especially from the reward-associated brain areas that project to the DMS (e.g., cortex, amygdala, and BNST). Interestingly, although dopamine receptors are widely distributed in the brain, the receptor subtype densities vary between different areas. A previous study discovered that D1R-expressing or D2R-expressing neurons were highly segregated in the orbitofrontal cortex, prefrontal cortex, and basolateral amygdala (Wei et al., 2018). However, the distribution pattern of D1R-expressing and D2R-expressing remains unclear in the BNST and CeA. The BNST and CeA play crucial roles in many neuropsychiatric disorders that include fear formation, anxiety, or reward-related impulsivity (Davis et al., 2010; Kim et al., 2018). Dopaminergic afferents from the ventral tegmental area innervate the amygdala and BNST, but the detailed D1R-expression and D2R-expression patterns remain unclear. Therefore, we crossed D1-tdTomato transgenic mice, which expressed tdTomato under the control of the 
D1R gene, with D2-Cre;Snap25 mice. We counted neurons expressing D1Rs (tdTomato-positive) and D2Rs (GFP-positive) in coronal sections containing the BNST and CeA of these D1-tdTomato;D2-Cre;Snap25 transgenic mice. Strong expression of GFP and tdTomato was observed in the striatum (Fig. $3 A, B$ ). However, while strong GFP expression was present in the BNST area, tdTomato expression was barely detectable (Fig. $3 A, B$ ). For each mouse, we examined six BNST sections from bregma AP +0.26 to $-0.34 \mathrm{~mm}$ (Fig. $3 C$ ). The average number of D1R-expressing neurons (41) was significantly lower than the average number of D2R-expressing neurons (215; $t_{(34)}=-3.28, p<0.01$; Fig. $\left.3 D\right)$.

A previous study showed relatively high $\mathrm{D} 1 \mathrm{R}$ expression and weak D2R expression in the basolateral amygdala (Wei et al., 2018). Interestingly, using the same transgenic animals, the present study identified a high level of D2R expression in the CeA (Fig. $3 E, F)$. We examined six CeA sections from bregma AP -0.82 to $-1.46 \mathrm{~mm}$ (Fig. $3 G$ ). An average of $82 \mathrm{D1R}$-expressing neurons was observed, and this value was significantly lower than the average number of D2R-expressing neurons $\left(439 ; t_{(34)}=-7.42, p<0.001\right.$; Fig. $\left.3 H\right)$. These results indicate that D2Rs are preferentially expressed in the BNST and CeA.

\section{Whole-brain mapping of D1R-expressing or D2R- expressing inputs to $\mathrm{PDMS}$ MSN subtypes}

The above results (Fig. 3) and recent transgenic models have revealed a tight segregation of D1Rs and D2Rs in some reward-related structures, including the orbitofrontal cortex, prefrontal cortex, and amygdala (Wei et al., 2018). In addition to the differential innervation of D1MSNs and D2-MSNs, it is essential to note that previous anatomic studies indicated that cortical fibers in the dorsal striatum contained abundant D2Rs, but few D1Rs (Wang and Pickel, 2002; Dumartin et al., 2007). In addition, a recent study reported that DMS-projecting extrastriatal neurons preferentially expressed D2Rs, rather than D1Rs (Lu et al., 2019). We thus asked whether extrastriatal D1R-expressing or D2R-expressing inputs showed biased projections to D1-MSNs or D2-MSNs in the pDMS. To test this, we measured the overlap between neurons that projected to D1MSNs or D2-MSNs in the pDMS, and extrastriatal neurons that expressed D1Rs or D2Rs. To explore the anatomic connections between extrastriatal D1R-expressing inputs and D1-MSNs (D1 $\rightarrow$ D1), and between D2R-expressing inputs and D2-MSNs (D2 $\rightarrow$ D2), we analyzed the numbers of neurons that were both GFP-positive and tdTomato-positive in D1-Cre;Snap25 and in D2-Cre;Snap25 mice following EnvA$\mathrm{SAD} \Delta \mathrm{G}-\mathrm{mCherry}$ injection.

D1R-expressing or D2R-expressing neurons were selectively labeled with GFP (Fig. $4 A, B, D$ left, $D$ middle) and the EnvA-SAD $\Delta$ G-mCherry rabies virus infected D1MSNs or D2-MSNs before spreading to neurons with direct inputs to these cells (Fig. $4 A, B, D$ left, $D$ middle). As a result, neurons that projected to D1-MSNs or D2-MSNs were labeled red (mCherry) in these mice. To probe the connections between extrastriatal D1R-expressing inputs and D2-MSNs (D1 $\rightarrow \mathrm{D} 2$ ), we employed D1-tdTomato;D2Cre mice, in which extrastriatal D1R-expressing neurons and pDMS D1-MSNs were both labeled red (tdTomato; Fig. 4C,D right). These mice did not express GFP in D2MSNs, and GFP was subsequently used in the rabies virus. Injection of AAV8-Flex-TVA-mCherry and AAV8Flex-RG into the pDMS, followed by EnvA-SAD $\Delta$ G-GFP, led to the infection of Cre-positive D2-MSNs and extrastriatal inputs to D2-MSNs by rabies virus; these expressed GFP and were thus labeled green (Fig. 4C,D right). Note that $\mathrm{pDMS}$-projecting neurons that contained D1Rs (or D2Rs) showed co-expression of mCherry (or tdTomato) and GFP and were thus yellow (Fig. 4D).

To our surprise, the majority of extrastriatal neurons that projected to D1-MSNs or D2-MSNs did not express D1Rs or D2Rs. Specifically, the D1R was expressed by $2.6 \pm 0.5 \%$ of the rabies virus-labeled neurons that projected to D1-MSNs in D1-Cre;Snap25 mice (D1 $\rightarrow$ D1), and the D2R was expressed by $5.4 \pm 0.9 \%$ of the inputs to D2-MSNs in D2-Cre;Snap25 mice (D2 $\rightarrow$ D2). The D1R was expressed by $0.8 \pm 0.4 \%$ of the inputs to D2-MSNs in D1-tdTomato;D2-Cre mice (D1 $\rightarrow \mathrm{D} 2)$. We directly compared the number of D1R-expressing neurons that project to the D1-MSNs (D1 $\rightarrow \mathrm{D} 1)$, D2R-expressing neurons that project to the D2-MSNs (D2 $\rightarrow \mathrm{D} 2$ ), and D1R-expressing neurons that project to the D2-MSNs $(\mathrm{D} 1 \rightarrow \mathrm{D} 2)$, and we found that there were significantly more $\mathrm{D} 2 \rightarrow \mathrm{D} 2$ connections than either $\mathrm{D} 1 \rightarrow \mathrm{D} 1$ or $\mathrm{D} 1 \rightarrow \mathrm{D} 2$ connections $(p<0.05$ and $p<0.01$, respectively; Fig. $4 E$ ). This was consistent with previous anatomic studies showing greater D2R expression, as compared with D1R expression, at presynaptic terminals in the dorsal striatum (Wang and Pickel, 2002; Dumartin et al., 2007; Lu et al., 2019).

Interestingly, although the number of extrastriatal D1Rexpressing or D2R-expressing neurons that projected to D1-MSNs or D2-MSNs was low, these neurons showed distinct distribution patterns across the brain. We normalized D1R-expressing or D2R-expressing inputs from individual brain regions to the total number of extrastriatal D1Rexpressing or D2R-expressing inputs. Distinct D1 $\rightarrow$ D1, $\mathrm{D} 2 \rightarrow \mathrm{D} 2$, and $\mathrm{D} 1 \rightarrow \mathrm{D} 2$ connections were observed in cortical and thalamic structures, but not in the amygdala $\left(F_{(2,20)}=\right.$ 60.22, $p<0.001$; Fig. 4F). Cortical regions had higher percentages of $\mathrm{D} 1 \rightarrow \mathrm{D} 1$ or $\mathrm{D} 1 \rightarrow \mathrm{D} 2$ connection, as compared with D2 $\rightarrow$ D2 $(p=0.001$; Fig. $4 F)$, while the thalamus had a higher percentage of $\mathrm{D} 2 \rightarrow \mathrm{D} 2$ than of $\mathrm{D} 1 \rightarrow \mathrm{D} 1$ or $\mathrm{D} 1 \rightarrow \mathrm{D} 2$ $(p<0.05$; Fig. $4 F)$. In the SNc, a higher percentage of the connections was $\mathrm{D} 2 \rightarrow \mathrm{D} 2$, as compared with $\mathrm{D} 1 \rightarrow \mathrm{D} 1$ or D1 $\rightarrow$ D2 $(p<0.01$; Fig. $4 G)$. In the BNST, a higher percentage of the connections was D2 $\rightarrow \mathrm{D} 2$, as compared with $\mathrm{D} 1 \rightarrow \mathrm{D} 2$ ( $p<0.05$; Fig. 4G).

We also analyzed the distribution of $\mathrm{D} 1 \rightarrow \mathrm{D} 1, \mathrm{D} 2 \rightarrow \mathrm{D} 2$, and $\mathrm{D} 1 \rightarrow \mathrm{D} 2$ in subregions of the cortex, amygdala, and thalamus that provided the most inputs to the pDMS. Analysis of ten cortical regions found a marginally higher percentage of $\mathrm{D} 1 \rightarrow \mathrm{D} 2$ than of $\mathrm{D} 2 \rightarrow \mathrm{D} 2$ projections from the frontal associate cortex $(p=0.082$; Fig. $5 A)$. D1 $\rightarrow$ D2 was also detected more frequently than $\mathrm{D} 2 \rightarrow \mathrm{D} 2$ from the cingulate cortex, although these differences did not achieve statistical significance $(p=0.074$; Fig. $5 A)$. The basolateral amygdala and $\mathrm{CeA}$ contained similar percentages of D1 $\rightarrow \mathrm{D} 1, \mathrm{D} 2 \rightarrow \mathrm{D} 2$, and $\mathrm{D} 1 \rightarrow \mathrm{D} 2$ connections 


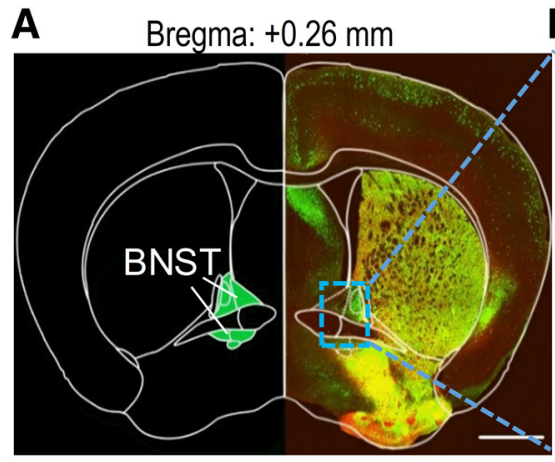

C

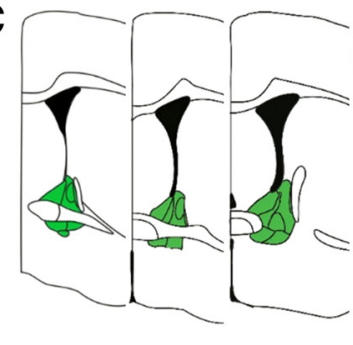

$+0.26+0.14+0.02$
$\mathrm{B}_{\mathrm{D}^{+} \mathrm{R}^{+}}$(GFP)
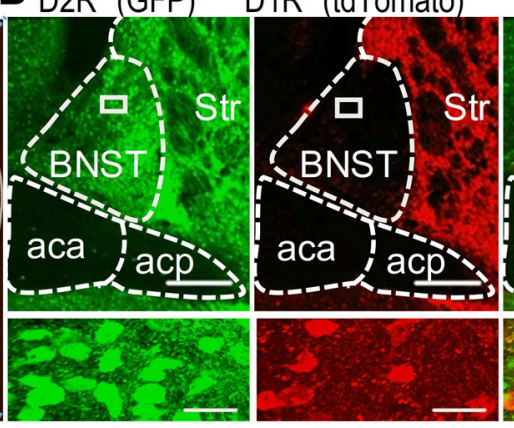

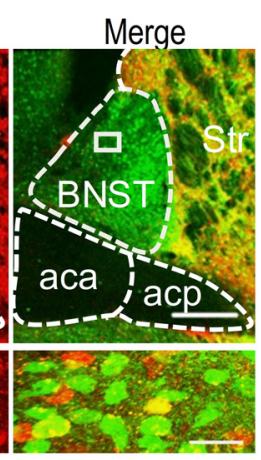

D

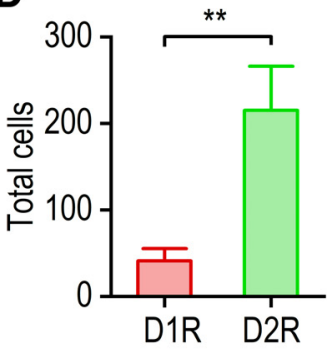

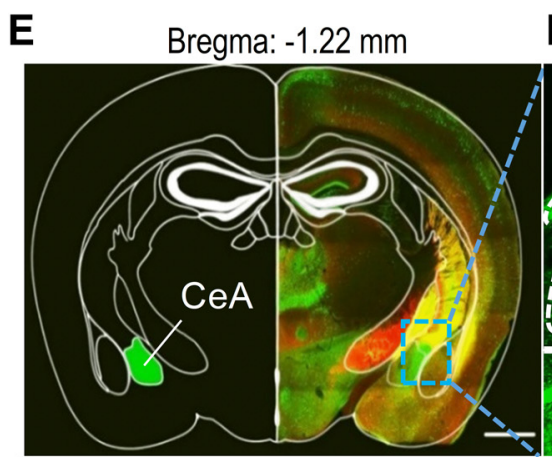

G

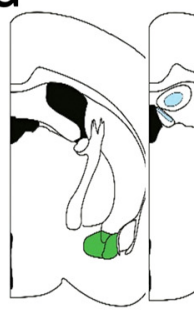

$-0.82$

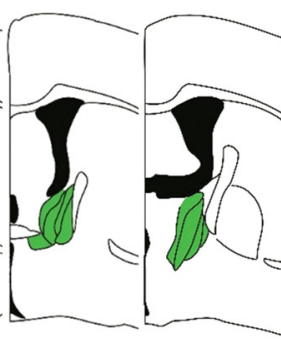

$-0.1$

$-0.22$

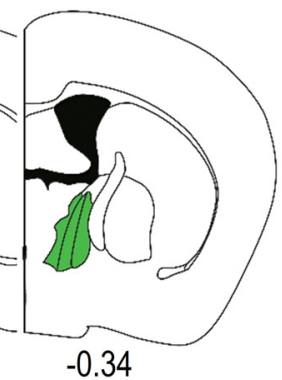

$\mathbf{F}_{\mathrm{D}^{2} \mathrm{R}^{+} \text {(GFP) }}$

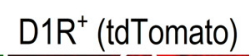

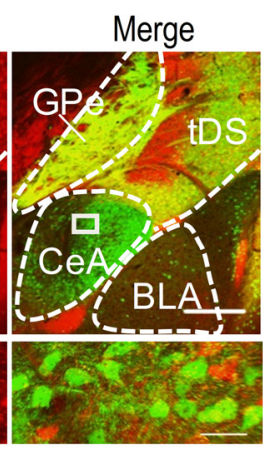

H

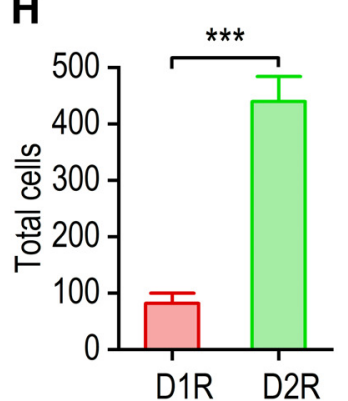

Figure 3. The BNST and CeA preferentially express D2Rs versus D1Rs. A, Representative fluorescent image of a coronal section of the BNST from a D1-tdTomato;D2-Cre;Snap25 mouse. The atlas skeleton (left) shows the BNST location at $+0.26 \mathrm{~mm}$ relative to bregma. $\boldsymbol{B}$, Representative dual-channel higher magnification fluorescent images of the boxed region of panel A showing abundant GFP-expressing (D2R-positive) neurons (left), a few tdTomato-expressing (D1R-positive) neurons (middle), and some colocalization (right). The bottom panels show an enlarged image of the boxed region from the top panels. Str, striatum; aca, anterior commissure area; acp, posterior commissure area. $\boldsymbol{C}$, Schematic representation of the BNST starting at $+0.26 \mathrm{~mm}$ and ending at $-0.34 \mathrm{~mm}$ relative to bregma. $\boldsymbol{D}$, Bar graph summarizing the numbers of D1R-expressing and D2R-expressing neurons in the BNST; ${ }^{* *} p<0.01$, unpaired $t$ test; $n=18$ sections from three mice. $\boldsymbol{E}$, Representative fluorescent image of a coronal section of the CeA from a D1-tdTomato;D2-Cre;Snap25 mouse. The atlas skeleton (left) shows the location of the $\mathrm{CeA}$ at $-1.22 \mathrm{~mm}$ relative to bregma. $\boldsymbol{F}$, Representative dual-channel higher magnification fluorescent images of the boxed region of panel E showing abundant GFP-expressing D2R-positive neurons (left), a few tdTomato-expressing D1R-positive neurons (middle), and some colocalization (right). The bottom panels show enlarged images of the boxed region of the top panels. GPe, globus pallidus external; tDS; tail of the striatum; BLA, basolateral amygdala. G, Schematic representation of the $\mathrm{CeA}$ starting at $-0.82 \mathrm{~mm}$ and ending at $-1.46 \mathrm{~mm}$ relative to bregma. $\boldsymbol{H}$, Bar graph depicting the higher average number of D2R-expressing neurons than D1R-expressing neurons in the CeA; ${ }^{* * *} p<0.001$, unpaired $t$ test; $n=18$ sections from three mice. Scale bars: 1 $\mathrm{mm}(\boldsymbol{A}, \boldsymbol{E}), 250 \mu \mathrm{m}(\boldsymbol{B}, \boldsymbol{F}$, top panels), $20 \mu \mathrm{m}$ ( $\boldsymbol{B}, \boldsymbol{F}$, bottom panels). 
A

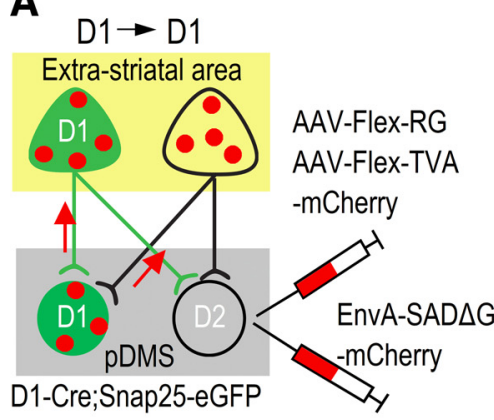

D

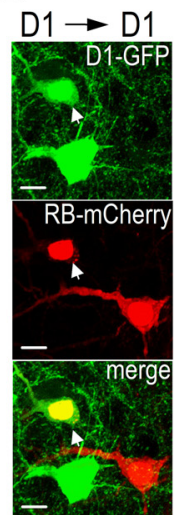

$G^{\text {D1-Cre;Snap25 }}$

G

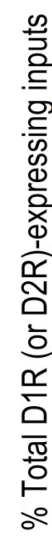

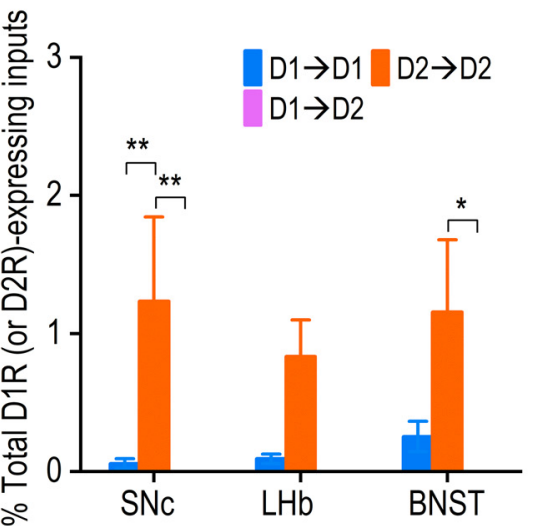

B

$$
\mathrm{D} 2 \rightarrow \mathrm{D} 2
$$

Extra-striatal area

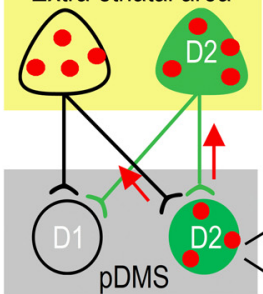

D2-Cre;Snap25-eGFP

E
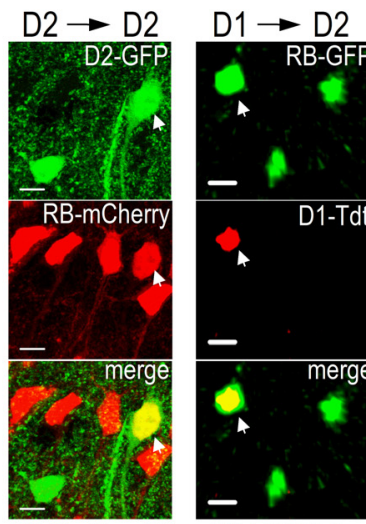

D1-tdT;D2-Cre

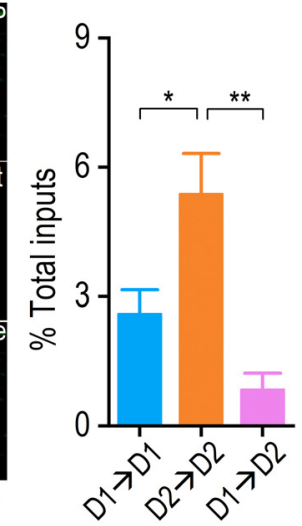
-mCherry
C

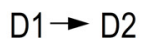

Extra-striatal area

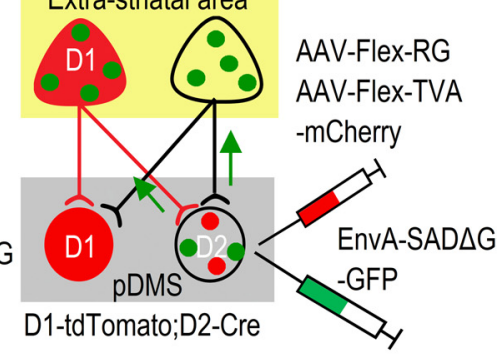

AAV-Flex-TVA

EnvA-SAD $\triangle \mathrm{G}$

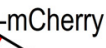

$F$

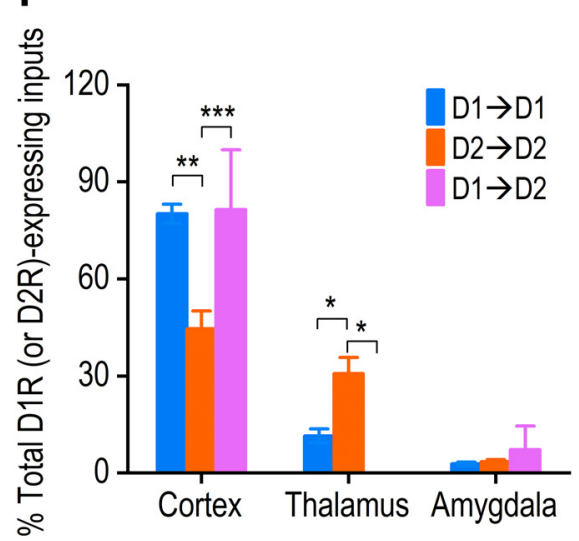

Figure 4. Rabies virus-mediated retrograde monosynaptic whole-brain labeling of D1R-expressing and D2R-expressing neurons projecting to D1-MSNs and D2-MSNs in the pDMS. $\boldsymbol{A}-\boldsymbol{C}$, Schematic showing the experimental approach used to label extrastriatal D1R-expressing or D2R-expressing neurons with projections to pDMS D1-MSNs or D2-MSNs. D1-Cre;Snap25 mice were employed to identify $\mathrm{D} 1 \rightarrow \mathrm{D} 1$ connections $(\boldsymbol{A})$, D2-Cre;Snap25 mice for D2 $\rightarrow$ D2 connections $(\boldsymbol{B})$, and D1-tdTomato;D2-Cre mice for D1 $\rightarrow$ D2 connections $(\boldsymbol{C})$. D1-Cre;Snap25 and D2-Cre;Snap25 mice expressed GFP in D1R-expressing and D2R-expressing neurons, respectively $(\boldsymbol{A}, \boldsymbol{B})$. In D1-tdTomato;D2-Cre mice, D1R-expressing neurons were labeled red (C). Injection of Cre-dependent helper viruses (AAV-Flex-TVA-mCherry and AAV-Flex-RG) into the pDMS induced selective expression of TVA and RG in Cre-expressing D1-MSNs $(\boldsymbol{A})$, D2-MSNs $(\boldsymbol{B})$, and D2-MSNs $(\boldsymbol{C})$. Three weeks after helper virus infusion, injection of EnvA-SAD $\triangle \mathrm{G}-\mathrm{mCherry}$ into the same site of the D1-Cre;Snap25 (A) and D2-Cre;Snap25 (B) mice, and of EnvA-SAD $\Delta$ G-GFP into the same site of D1-tdTomato;D2Cre mice $(\boldsymbol{C})$ caused selective rabies infection and expression of mCherry by D1-MSNs $(\boldsymbol{A})$ or D2-MSNs $(\boldsymbol{B})$, and expression of rabies-GFP by D2-MSNs $(\boldsymbol{C})$. Retrograde spread of rabies virus then occurred from D1-MSNs $(\boldsymbol{A})$ or D2-MSNs $(\boldsymbol{B}, \boldsymbol{C})$ to extrastriatal presynaptic neurons. This facilitated identification of $\mathrm{D} 1 \rightarrow \mathrm{D} 1(\boldsymbol{A}), \mathrm{D} 2 \rightarrow \mathrm{D} 2(\boldsymbol{B})$, and $\mathrm{D} 1 \rightarrow \mathrm{D} 2(\boldsymbol{C})$ connections, as indicated. $\boldsymbol{D}$, Representative confocal images of the cortex in the indicated mice following injection of either EnvA-SAD $\Delta$ G-mCherry or EnvA$\mathrm{SAD} \Delta \mathrm{G}$-GFP. White arrows indicate colocalization of D1 $\rightarrow \mathrm{D} 1$ (left), D2 $\rightarrow \mathrm{D} 2$ (middle), and D1 $\rightarrow \mathrm{D} 2$ (right). Scale bars: $10 \mu \mathrm{m}$. $\boldsymbol{E}$, The relative levels of the indicated connections, where $\mathrm{D} 1 \rightarrow \mathrm{D} 1$ is expressed as a percentage of the total number of D1-MSN inputs, and $\mathrm{D} 2 \rightarrow \mathrm{D} 2$ or D1 $\rightarrow \mathrm{D} 2$ is expressed as a percentage of the total number of D2-MSN inputs; ${ }^{*} p<0.05$, ${ }^{* *} p<0.01$ for the indicated comparisons, one-way ANOVA followed by Tukey's test. $\boldsymbol{F}$, Bar graph comparing D1R-expressing and D2R-expressing inputs from the cortex, amygdala, and thalamus onto MSNs. D1R-expressing or D2R-expressing inputs from the indicated brain regions were normalized to the total extrastriatal D1R-expressing or D2R-expressing inputs, as appropriate. Note that the cortex exhibited high 
continued

percentages of $\mathrm{D} 1 \rightarrow \mathrm{D} 1$ and $\mathrm{D} 1 \rightarrow \mathrm{D} 2$ connections, whereas the thalamus showed a high percentage of D2 $\rightarrow \mathrm{D} 2$ connections; ${ }^{* \star *} p<0.001,{ }^{\star} p<0.05$, two-way RM ANOVA followed by Tukey's test. G, Bar graph comparing D1R-expressing and D2R-expressing inputs from the SNc, lateral habenula (LHb), and BNST onto MSNs. D1R-expressing or D2R-expressing inputs from these brain regions were normalized to the total extrastriatal D1R-expressing or D2R-expressing inputs; ${ }^{* *} p<0.01,{ }^{\star} p<0.05$, two-way RM ANOVA followed by Tukey's test; $n=5$ (D1 $\rightarrow \mathrm{D} 1), n=4$ (D2 $\rightarrow \mathrm{D} 2), n=4$ (D1 $\rightarrow \mathrm{D} 2)$ mice $(\boldsymbol{E}-\mathrm{G})$.

$\left(F_{(1,10)}=0.62, p>0.05 ;\right.$ Fig. $\left.5 B\right)$. The data presented in Figure $5 C$ showed that a significantly higher percentage of $\mathrm{D} 2 \rightarrow \mathrm{D} 2$ than of $\mathrm{D} 1 \rightarrow \mathrm{D} 1$ or $\mathrm{D} 1 \rightarrow \mathrm{D} 2$ projections arose from the mediodorsal thalamic nucleus $(p<0.001)$, central thalamic nucleus $(p<0.001)$, and ventromedial thalamic nucleus $(p<0.001)$. A higher percentage of $D 1 \rightarrow D 1$ than of $\mathrm{D} 1 \rightarrow \mathrm{D} 2$ was observed in the parafascicular thalamic nucleus $(p<0.05$; Fig. $5 C)$. Together, these results suggest that while the number of D1R-expressing or D2R-expressing neurons with projections to pDMS MSNs was low, they exhibited distinct brain distribution patterns.

\section{Discussion}

Using the rabies virus-mediated retrograde monosynaptic tracing system, the present study investigated the inputs onto pDMS D1-MSNs and D2-MSNs and examined the distribution pattern of brain-wide extrastriatal D1R-expressing and D2R-expressing inputs to these MSNs. We found that the cortex, including the secondary motor and cingulate cortices, preferentially projects to pDMS D1-MSNs, whereas the thalamus, including the central and ventromedial thalamic nuclei, preferentially innervates pDMS D2-MSNs. In addition, while providing fewer inputs than the cortex and thalamus to the pDMS, the BNST and CeA contained more D2R-expressing than D1R-expressing neurons. Lastly, we discovered that MSN-projecting neurons exhibited distinct distribution patterns of the D1R and D2R across the whole brain. These results suggest that the segregation of connections between upstream brain regions and pDMS D1-MSNs or D2-MSNs may provide the basis for biased information

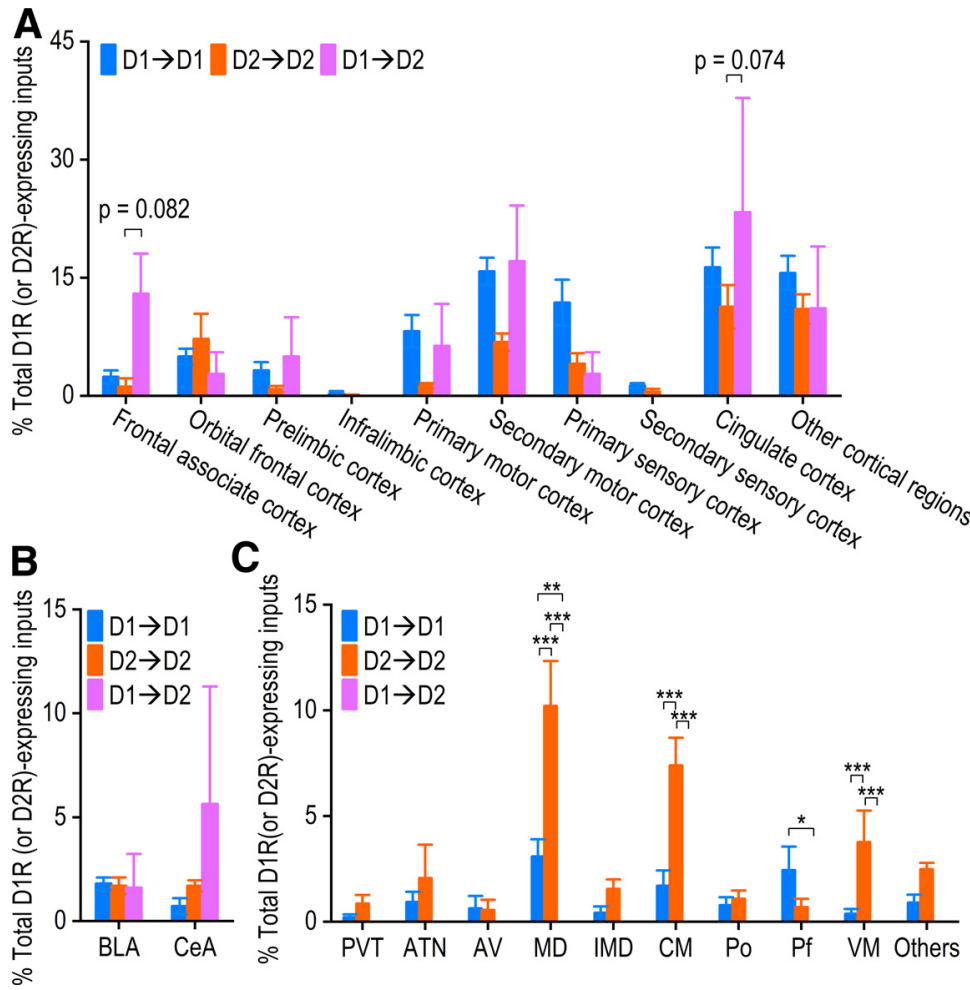

Figure 5. Distribution of extrastriatal D1R-expressing or D2R-expressing inputs onto MSNs from the cortex, amygdala, and thalamus. D1R-expressing or D2R-expressing inputs from the indicated brain regions were normalized to the total extrastriatal D1R-expressing or D2R-expressing inputs, as appropriate. $\boldsymbol{A}$, Bar graph of cortical inputs, segregated into ten major subregions, indicating that the frontal associate cortex had a high proportion of D1 $\rightarrow \mathrm{D} 2$ connections; * $p<0.05$, two-way RM ANOVA followed by Tukey's test. $\boldsymbol{B}$, No significant differences were found in the proportions of connection types between the BLA or CeA and the pDMS. $\boldsymbol{C}$, No significant differences were found in the proportions of connection types between the paraventricular thalamic nucleus (PVT), anterior thalamic nucleus (ATN), anteroventral thalamic nucleus (AV), intermediodorsal thalamic nucleus (IMD), or posterior thalamic nucleus (Po) and the pDMS. However, the mediodorsal thalamic nucleus (MD), central thalamic nucleus (CM), and ventromedial thalamic nucleus (VM) were found to have high percentages of D2 $\rightarrow$ D2 connections, while the parafascicular thalamic nucleus (Pf) had a high percentage of D1 $\rightarrow \mathrm{D} 1$ connections; ${ }^{* *} p<0.001,{ }^{* *} p<0.01,{ }^{*} p<0.05$, two-way RM ANOVA followed by Tukey's test; $n=5(\mathrm{D} 1 \rightarrow \mathrm{D} 1), n=4(\mathrm{D} 2 \rightarrow \mathrm{D} 2), n=4(\mathrm{D} 1 \rightarrow \mathrm{D} 2)$ mice. 
propagation to basal ganglia structures, resulting in differential effects on behavior. In addition, D2R-expressing neurons in the BNST and CeA may exert distinct roles in anxiety and addiction.

\section{Distinct monosynaptic inputs to pDMS D1-MSNs and D2-MSNs}

Given the broad roles of the striatum in motor execution, action selection, learning, behavioral flexibility, and reinforcement-associated behaviors such as rewardseeking and reinstatement (Ragozzino ME et al., 2002; Bamford et al., 2018; Roltsch Hellard et al., 2019), improving our understanding of how individual brain regions precisely innervate two major subtypes of striatal neurons represent an essential step toward dissecting the circuits that impact striatal function. The striatum extends a significant length along the anterior-posterior axis, and growing evidence (Yin and Knowlton, 2004; Yin et al., 2005a,b; Corbit and Janak, 2010; Shiflett et al., 2010; Regier et al., 2015) suggests a functional dissociation between anterior and posterior striatum. Although the coordinates that have been used for defining anterior and posterior of striatum varies, the coronal sections (e.g., $-0.4 \mathrm{~mm}$ ) posterior to the anterior commissure crossing the midline were usually considered as the posterior striatum in the majority of rat studies (Betancur et al., 1997; Yin and Knowlton, 2004; Yin et al., 2005a,b; Lex and Hauber, 2010; Shiflett et al., 2010; Stalnaker et al., 2010; Bradfield et al., 2013; Corbit et al., 2013; Hart and Balleine, 2016). According to the Paxinos atlas (Franklin and Paxinos, 2007), the anterior commissure crosses the midline more anteriorly in the mouse brain (at $+0.14 \mathrm{~mm}$ ) than in the rat brain (at -0.12 $\mathrm{mm}$ ). Thus, a section at $0.0 \mathrm{~mm}$ from bregma in a mouse brain was used to define the posterior DMS (Shan et al., 2014; Matamales et al., 2016; Wang et al., 2018). Note that increasing evidence indicate that a third striatal portion along the anterior-posterior axis, the tail of the striatum, exhibits distinct functions and anatomic connections from aDMS or pDMS (Menegas et al., 2015, 2017, 2018; Matamales et al., 2016; Guo et al., 2018, 2019; Gangarossa et al., 2019).

The present study discovered that pDMS D1-MSNs and D2-MSNs received asymmetric inputs from other brain regions. We employed a monosynaptic rabies virus system (Wall et al., 2013) to label brain-wide neurons with direct connections to pDMS D1-MSNs or D2-MSNs. We discovered that these two MSN subtypes received asymmetric inputs from other whole brain regions. Dense D1MSN innervations originated primarily from the orbital frontal, secondary motor, cingulate, and secondary visual cortices. These prefrontal and limbic structures are devoted mainly to reward motivation, emotional regulation, planning of complex cognitive behaviors, and decisionmaking (Floresco et al., 2008; Balleine et al., 2009; Gremel et al., 2016; Barthas and Kwan, 2017). Therefore, our results suggested that information related to reward, based on past experience, may be preferentially passed to the direct pathway circuit and thus facilitate actions likely to procure a reward. In contrast, D2-MSNs received inputs that mainly originated from the primary motor cortex, primary sensory cortex, central thalamic nucleus, and ventromedial thalamic nucleus. Concurrent activation of D1-MSNs and D2-MSNs has been reported during action initiation (Hikida et al., 2010; Cui et al., 2013). Biased projections from the primary motor and sensory cortices onto D2-MSNs may contribute to the suppression of unwanted behavior via activation of D2-MSNs. In addition, there is growing evidence that the thalamostriatal pathway plays an essential role in responses to salient stimuli and behavioral flexibility; projections from the central median and parafascicular nuclei onto cholinergic interneurons, which have also been found to express the D2R, appear to be particularly important in this context (Kreitzer and Malenka, 2008; Smith et al., 2011; Bradfield et al., 2013; Guo et al., 2015). The present study used D2-Cre mice to identify extrastriatal inputs onto D2-MSNs. Despite the relatively low abundance of striatal cholinergic interneurons (Kreitzer and Malenka, 2008), we cannot rule out the possibility that the observed connections between central and ventromedial thalamic regions and D2-expressing striatal neurons may also have included some projections to cholinergic interneurons. This architecture suggests that salient information from the thalamus may be differentially transmitted to D2R-expressing cholinergic interneurons and D2-MSNs to stop a current ongoing action and initiate a new action, thus facilitating flexible switching between behaviors.

The pDMS appears to be more involved in the acquisition of goal-directed actions than the aDMS (Yin et al., 2005a; Corbit and Janak, 2010; Shiflett et al., 2010), and the present study, therefore, focused on inputs to the pDMS. Our results were mostly consistent with other recent studies, which identified major inputs onto D1-MSNs and D2-MSNs that arose in the cortex and thalamus (Doig et al., 2010; Wall et al., 2013; Hunnicutt et al., 2016). Interestingly, in contrast to a previous observation (Wall et al., 2013), we identified significant projections from the secondary motor cortex and cingulate cortex to the pDMS. These inputs selectively innervated D1-MSNs. Anatomical and functional studies have suggested that the dorsomedial, dorsolateral, and ventral striatum received preferential inputs from the associative, sensorimotor, and limbic structures, respectively (Burton et al., 2015; Hunnicutt et al., 2016). Thus, this discrepancy may reflect the medial injection site employed in the present study.

\section{Distinct expression of D1Rs and D2Rs in the BNST and CeA}

Interestingly, we observed that D2R-expressing neurons predominated in the BNST and CeA, which are closely associated with anxiety and addictive disorders (Davis et al., 2010; Kim et al., 2018). This was consistent with previous anatomic studies that identified a high proportion of D2R-expressing cells in the BNST and CeA using D2-GFP transgenic animals and D2 in situ hybridization (Perez de la Mora et al., 2012; De Bundel et al., 2016). The CeA and BNST receive dense dopaminergic inputs that originate in the ventral tegmental area (Hasue and Shammah-Lagnado, 2002; Krawczyk et al., 2011). A 
study using a mouse behavioral paradigm for auditory threat response generalization found that dopamine facilitated the consolidation of fear memory through concomitant activation of D2Rs in the CeA and BNST (De Bundel et al., 2016). A previous immunohistochemical study revealed that most D2R-expressing cells were also positive for protein kinase C- $\delta$ (Kim et al., 2018), a marker previously shown to identify GABAergic neurons. Selective expression of D2Rs in the BNST and CeA may have a critical impact on final behavioral outcomes in anxiety-related and reward-related processes.

\section{D1R-expressing or D2R-expressing inputs to D1-MSNs and D2-MSNs in the pDMS}

Dopaminergic modulation of corticostriatal neurotransmission is particularly crucial during reward-based behaviors (Lüscher and Malenka, 2011; Bamford et al., 2018; $\mathrm{Ma}$ et al., 2018). A recent anatomic study observed high expression levels of D1Rs and D2Rs in the medial prefrontal cortex, orbital frontal cortex, and amygdala (Wei et al., 2018). Furthermore, these D1R-expressing and D2R-expressing neurons were highly segregated, with a low percentage of neurons co-expressing both D1Rs and D2Rs (Wei et al., 2018). Importantly, we found that D1R-expressing or D2R-expressing neurons within the cortex and amygdala sent projections to the DMS and formed functional connections with D1-MSNs or D2-MSNs (Lu et al., 2019). Thus, we expected that there would be strong anatomic connections between extrastriatal D1R-expressing or D2R-expressing DMS-projecting neurons and D1-MSNs or D2-MSNs. Surprisingly, we did not detect a high level of overlap between extrastriatal neurons expressing D1Rs or D2Rs and D1-MSNs or D2-MSNs; this suggested that the majority of extrastriatal neurons projecting to D1-MSNs or D2-MSNs did not express these receptors. Although only a minority of neurons with inputs to D1-MSNs or D2-MSNs expressed D1Rs or D2Rs, increasing evidence indicates that presynaptic D1Rs and D2Rs are essential for dopamine-dependent modulation of glutamate release at corticostriatal synapses (Wang and Goldman-Rakic, 2004; Bamford et al., 2018; Cui et al., 2018; Lu et al., 2019).

When the cortical inputs are stimulated at a low frequency, activation of presynaptic D1Rs was shown to boost glutamate release to D1 and D2-MSNs, whereas activation of presynaptic D2Rs suppressed glutamate release (Wang et al., 2012; Cui et al., 2018; Lu et al., 2019). However, when the cortical inputs are stimulated at a higher frequency, this dopaminergic modulation of glutamate release was blocked by adenosine and endocannabinoids (Bamford et al., 2018). This type of presynaptic dopaminergic modulation facilitates the appropriate selection and transmission of excitatory inputs via corticostriatal synapses during learning (Bamford et al., 2018). The D1R-expressing and D2R-expressing inputs to D1MSNs or D2-MSNs may play important roles in this presynaptic filtration.

We observed that D1R-expressing or D2R-expressing neurons with inputs to pDMS D1-MSNs or D2-MSNs had distinct brain distribution patterns. Cortical regions contained a higher percentage of $\mathrm{D} 1 \rightarrow \mathrm{D} 1$ or $\mathrm{D} 1 \rightarrow \mathrm{D} 2$ connections, as compared with $\mathrm{D} 2 \rightarrow \mathrm{D} 2$, while thalamic areas had more D2 $\rightarrow$ D2 connections, as compared with either $\mathrm{D} 1 \rightarrow \mathrm{D} 1$ or $\mathrm{D} 1 \rightarrow \mathrm{D} 2$. It is known that D1Rs exhibit a relatively low affinity for dopamine and are mainly activated by fast phasic release of high concentrations of dopamine, while D2Rs have a higher affinity for dopamine and mainly respond to slow tonic dopamine release (Richfield EK et al., 1989; Grieder et al., 2012). The relative levels of D1R-expressing and D2R-expressing inputs to MSNs from cortical and thalamic regions observed in this study suggest that the corticostriatal pathway may be more sensitive to phasic dopamine release. In contrast, the thalamostriatal pathway would be modulated by an increased basal dopamine level. These anatomic findings indicate that phasic dopamine release would preferentially initiate an action, while an increased basal dopamine level would stop an action. In addition, the SNc, lateral habenula, and BNST were similar to thalamic regions in that they expressed high levels of D2Rs than of D1Rs (Krawczyk et al., 2011; Ford, 2014). As we did not examine the anatomic connections between D2R-expressing inputs and D1-MSNs in the present study, it is not surprising that D2 $\rightarrow$ D2 connections were more prevalent than either $\mathrm{D} 1 \rightarrow \mathrm{D} 1$ or $\mathrm{D} 1 \rightarrow \mathrm{D} 2$ in these regions.

Interestingly, the analysis of brain-wide inputs onto pDMS D1-MSNs and D2-MSNs revealed that orbital frontal cortex, secondary motor cortex, cingulate cortex, and secondary visual cortex preferentially innervated D1-MSNs, whereas the primary motor cortex, primary sensory cortex, central thalamic nucleus, ventromedial thalamic nucleus, and globus pallidus preferentially innervated D2-MSNs (Fig. 2). Besides geometric subdivisions, our study also included cell-type-specific inputs, based on their expression of D1Rs or D2Rs, onto either type of MSNs. We found that extrastriatal D1R-expressing or D2R-expressing inputs also showed biased projections to D1-MSNs or D2-MSNs in the pDMS (Fig. 5). It is worth noting that the subregion-based distinct input connectivity of D1-MSNs or D2-MSNs does not guarantee all types of neurons from this region show the same innervation preference. For instance, we did not find biased projection onto D1-MSNs versus D2-MSNs from the frontal associated cortex in Figure 2. However, we observed that D1R-expressing neurons in this brain region preferentially innervated D2-MSNs versus D1-MSNs in Figure 5. Thus, the innervation segregation of MSNs is not clear-cut based on the classification of brain structures. Therefore, it is of particular interest to understand how individual cell-type-specific inputs from different subregions precisely innervate the D1MSNs and D2-MSNs.

\section{Technical consideration}

The modified rabies virus-mediated monosynaptic tracing enables cell-specific tracing and has been a powerful tool to reveal brain circuitry. However, this method has some technical limitations. The variability of viral expression or imaging conditions may contribute to the discovered differences in the relative proportion of rabies-labeled input cells from certain brain areas onto either D1-MSNs or D2-MSNs. In addition, 
the efficiency of retrograde transmission of rabies virus at different excitatory, inhibitory, and neuromodulatory synapses is still not fully understood. We and others observed that the dopamine neurons innervating D1MSNs or D2-MSNs only account for a small percentage of total neurons labeled by rabies virus (Wall et al., 2013; Smith et al., 2016), but the density of midbrain dopaminergic terminals and dopamine receptor expression were high within the striatum (Gerfen and Surmeier, 2011; Cheng et al., 2017; Wei et al., 2018). As compared with the corticostriatal excitatory synapses, those neuromodulatory synapses seem to be underestimated by rabies-mediated retrograde tracing. Similarly, the inhibitory synapses between BNST or CeA may be underreported by current data, as a strong inhibitory functional connection has been found from BNST to MSNs (Smith et al., 2016).

In summary, we have demonstrated that pDMS D1MSNs and D2-MSNs received differential innervation from cortical and thalamic structures. Additionally, we found that the majority of brain-wide extrastriatal inputs to D1-MSNs or D2-MSNs did not express D1Rs or D2Rs, and that the input neurons that did express these receptors exhibited distinct distribution patterns. These findings provide a foundation for the understanding of information segregation in pDMS circuits that will guide future studies.

\section{References}

Balleine BW, Liljeholm M, Ostlund SB (2009) The integrative function of the basal ganglia in instrumental conditioning. Behav Brain Res 199:43-52.

Bamford NS, Wightman RM, Sulzer D (2018) Dopamine's effects on corticostriatal synapses during reward-based behaviors. Neuron 97:494-510.

Barthas F, Kwan AC (2017) Secondary motor cortex: where 'sensory' meets 'motor' in the rodent frontal cortex. Trends Neurosci 40:181-193.

Betancur C, Rostène W, Bérod A (1997) Chronic cocaine increases neurotensin gene expression in the shell of the nucleus accumbens and in discrete regions of the striatum. Brain Res Mol Brain Res 44:334-340.

Bradfield LA, Bertran-Gonzalez J, Chieng B, Balleine BW (2013) The thalamostriatal pathway and cholinergic control of goal-directed action: interlacing new with existing learning in the striatum. Neuron 79:153-166.

Burton AC, Nakamura K, Roesch MR (2015) From ventral-medial to dorsal-lateral striatum: neural correlates of reward-guided decision-making. Neurobiol Learn Mem 117:51-59.

Cheng Y, Huang CC, Ma T, Wei X, Wang X, Lu J, Wang J (2017) Distinct synaptic strengthening of the striatal direct and indirect pathways drives alcohol consumption. Biol Psychiatry 81:918929.

Cheng Y, Wang X, Wei X, Xie X, Melo S, Miranda RC, Wang J (2018) Prenatal exposure to alcohol induces functional and structural plasticity in dopamine D1 receptor-expressing neurons of the dorsomedial striatum. Alcohol Clin Exp Res 42:1493-1502.

Corbit LH, Janak PH (2010) Posterior dorsomedial striatum is critical for both selective instrumental and Pavlovian reward learning. Eur J Neurosci 31:1312-1321.

Corbit LH, Leung BK, Balleine BW (2013) The role of the amygdalastriatal pathway in the acquisition and performance of goal-directed instrumental actions. J Neurosci 33:17682-17690.
Cui G, Jun SB, Jin X, Pham MD, Vogel SS, Lovinger DM, Costa RM (2013) Concurrent activation of striatal direct and indirect pathways during action initiation. Nature 494:238-242.

Cui Q, Li Q, Geng H, Chen L, Ip NY, Ke Y, Yung WH (2018) Dopamine receptors mediate strategy abandoning via modulation of a specific prelimbic cortex-nucleus accumbens pathway in mice. Proc Natl Acad Sci USA 115:E4890-E4899.

Davis M, Walker DL, Miles L, Grillon C (2010) Phasic vs sustained fear in rats and humans: role of the extended amygdala in fear vs anxiety. Neuropsychopharmacology 35:105-135.

De Bundel D, Zussy C, Espallergues J, Gerfen CR, Girault JA, Valjent E (2016) Dopamine D2 receptors gate generalization of conditioned threat responses through mTORC1 signaling in the extended amygdala. Mol Psychiatry 21:1545-1553.

Doig NM, Moss J, Bolam JP (2010) Cortical and thalamic innervation of direct and indirect pathway medium-sized spiny neurons in mouse striatum. J Neurosci 30:14610-14618.

Dumartin B, Doudnikoff E, Gonon F, Bloch B (2007) Differences in ultrastructural localization of dopaminergic D1 receptors between dorsal striatum and nucleus accumbens in the rat. Neurosci Lett 419:273-277.

Floresco SB, St Onge JR, Ghods-Sharifi S, Winstanley CA (2008) Cortico-limbic-striatal circuits subserving different forms of costbenefit decision making. Cogn Affect Behav Neurosci 8:375-389.

Ford CP (2014) The role of D2-autoreceptors in regulating dopamine neuron activity and transmission. Neuroscience 282:13-22.

Franklin KBJ, Paxinos G (2007) The mouse brain in stereotaxic coordinates, Ed 3. New York: Elsevier.

Gangarossa G, Castell L, Castro L, Tarot P, Veyrunes F, Vincent P, Bertaso F, Valjent E (2019) Contrasting patterns of ERK activation in the tail of the striatum in response to aversive and rewarding signals. J Neurochem 151:204-226.

Gerfen CR, Surmeier DJ (2011) Modulation of striatal projection systems by dopamine. Annu Rev Neurosci 34:441-466.

Gerfen CR, Paletzki R, Heintz N (2013) GENSAT BAC Cre-recombinase driver lines to study the functional organization of cerebral cortical and basal ganglia circuits. Neuron 80:1368-1383.

Gremel CM, Chancey JH, Atwood BK, Luo G, Neve R, Ramakrishnan C, Deisseroth K, Lovinger DM, Costa RM (2016) Endocannabinoid modulation of orbitostriatal circuits gates habit formation. Neuron 90:1312-1324.

Grieder TE, George O, Tan H, George SR, Le Foll B, Laviolette SR, van der Kooy D (2012) Phasic D1 and tonic D2 dopamine receptor signaling double dissociate the motivational effects of acute nicotine and chronic nicotine withdrawal. Proc Natl Acad Sci USA 109:3101-3106.

Guo L, Walker WI, Ponvert ND, Penix PL, Jaramillo S (2018) Stable representation of sounds in the posterior striatum during flexible auditory decisions. Nat Commun 9:1534.

Guo L, Weems JT, Walker WI, Levichev A, Jaramillo S (2019) ChoiceSelective Neurons in the Auditory Cortex and in Its Striatal Target Encode Reward Expectation. J Neurosci 39:3687-3697.

Guo Q, Wang D, He X, Feng Q, Lin R, Xu F, Fu L, Luo M (2015) Whole-brain mapping of inputs to projection neurons and cholinergic interneurons in the dorsal striatum. PLoS One 10:e0123381.

Hart G, Balleine BW (2016) Consolidation of goal-directed action depends on MAPK/ERK signaling in rodent prelimbic cortex. J Neurosci 36:11974-11986.

Hasue RH, Shammah-Lagnado SJ (2002) Origin of the dopaminergic innervation of the central extended amygdala and accumbens shell: a combined retrograde tracing and immunohistochemical study in the rat. J Comp Neurol 454:15-33.

Hernández VM, Hegeman DJ, Cui Q, Kelver DA, Fiske MP, Glajch KE, Pitt JE, Huang TY, Justice NJ, Chan CS (2015) Parvalbumin+ neurons and npas1+ neurons are distinct neuron classes in the mouse external globus pallidus. J Neurosci 35:11830-11847.

Hikida T, Kimura K, Wada N, Funabiki K, Nakanishi S (2010) Distinct roles of synaptic transmission in direct and indirect striatal pathways to reward and aversive behavior. Neuron 66:896-907. 
Huang CCY, Ma T, Roltsch Hellard EA, Wang X, Selvamani A, Lu J, Sohrabji F, Wang J (2017) Stroke triggers nigrostriatal plasticity and increases alcohol consumption in rats. Sci Rep 7:2501.

Hunnicutt BJ, Jongbloets BC, Birdsong WT, Gertz KJ, Zhong H, Mao $\mathrm{T}$ (2016) A comprehensive excitatory input map of the striatum reveals novel functional organization. Elife 5:e19103.

Kim B, Yoon S, Nakajima R, Lee HJ, Lim HJ, Lee YK, Choi JS, Yoon BJ, Augustine GJ, Baik JH (2018) Dopamine D2 receptor-mediated circuit from the central amygdala to the bed nucleus of the stria terminalis regulates impulsive behavior. Proc Natl Acad Sci USA 115:E10730-E10739.

Krawczyk M, Georges F, Sharma R, Mason X, Berthet A, Bézard E, Dumont EC (2011) Double-dissociation of the catecholaminergic modulation of synaptic transmission in the oval bed nucleus of the stria terminalis. J Neurophysiol 105:145-153.

Kreitzer AC, Malenka RC (2008) Striatal plasticity and basal ganglia circuit function. Neuron 60:543-554.

Lee CR, Abercrombie ED, Tepper JM (2004) Pallidal control of substantia nigra dopaminergic neuron firing pattern and its relation to extracellular neostriatal dopamine levels. Neuroscience 129:481489.

Lex B, Hauber W (2010) Disconnection of the entorhinal cortex and dorsomedial striatum impairs the sensitivity to instrumental contingency degradation. Neuropsychopharmacology 35:17881796.

Lovinger DM (2010) Neurotransmitter roles in synaptic modulation, plasticity and learning in the dorsal striatum. Neuropharmacology 58:951-961.

Lu J, Cheng Y, Wang X, Woodson K, Kemper C, Disney E, Wang J (2019) Alcohol intake enhances glutamatergic transmission from D2 receptor-expressing afferents onto D1 receptor-expressing medium spiny neurons in the dorsomedial striatum. Neuropsychopharmacology 44:1123-1131.

Lüscher C, Malenka RC (2011) Drug-evoked synaptic plasticity in addiction: from molecular changes to circuit remodeling. Neuron 69:650-663.

Lüscher C, Robbins TW, Everitt BJ (2020) The transition to compulsion in addiction. Nat Rev Neurosci 21:247-263.

Ma T, Barbee B, Wang X, Wang J (2017) Alcohol induces input-specific aberrant synaptic plasticity in the rat dorsomedial striatum. Neuropharmacology 123:46-54.

Ma T, Cheng Y, Roltsch Hellard E, Wang X, Lu J, Gao X, Huang CCY, Wei X, Ji J, Wang J (2018) Bidirectional and long-lasting control of alcohol-seeking behavior by corticostriatal LTP and LTD. Nat Neurosci 21:373-383.

Madisen L, Garner AR, Shimaoka D, Chuong AS, Klapoetke NC, Li L, van der Bourg A, Niino Y, Egolf L, Monetti C, Gu H, Mills M, Cheng A, Tasic B, Nguyen TN, Sunkin SM, Benucci A, Nagy A, Miyawaki A, Helmchen F, et al. (2015) Transgenic mice for intersectional targeting of neural sensors and effectors with high specificity and performance. Neuron 85:942-958.

Matamales M, Skrbis Z, Hatch RJ, Balleine BW, Götz J, BertranGonzalez J (2016) Aging-related dysfunction of striatal cholinergic interneurons produces conflict in action selection. Neuron 90:362373.

Menegas W, Bergan JF, Ogawa SK, Isogai Y, Umadevi Venkataraju K, Osten P, Uchida N, Watabe-Uchida M (2015) Dopamine neurons projecting to the posterior striatum form an anatomically distinct subclass. Elife 4:e10032.

Menegas W, Babayan BM, Uchida N, Watabe-Uchida M (2017) Opposite initialization to novel cues in dopamine signaling in ventral and posterior striatum in mice. Elife 6:e21886.

Menegas W, Akiti K, Amo R, Uchida N, Watabe-Uchida M (2018) Dopamine neurons projecting to the posterior striatum reinforce avoidance of threatening stimuli. Nat Neurosci 21:1421-1430.

Ogawa SK, Watabe-Uchida M (2018) Organization of dopamine and serotonin system: anatomical and functional mapping of monosynaptic inputs using rabies virus. Pharmacol Biochem Behav 174:9-22.
Perez de la Mora M, Gallegos-Cari A, Crespo-Ramirez M, Marcellino D, Hansson AC, Fuxe K (2012) Distribution of dopamine $D(2)$-like receptors in the rat amygdala and their role in the modulation of unconditioned fear and anxiety. Neuroscience 201:252-266.

Ragozzino ME, Ragozzino KE, Mizumori SJ, Kesner RP (2002) Role of the dorsomedial striatum in behavioral flexibility for response and visual cue discrimination learning. Behav Neurosci 116:105115.

Regier PS, Amemiya S, Redish AD (2015) Hippocampus and subregions of the dorsal striatum respond differently to a behavioral strategy change on a spatial navigation task. J Neurophysiol 114:1399-1416.

Richfield EK, Penney JB, Young AB (1989) Anatomical and affinity state comparisons between dopamine D1 and D2 receptors in the rat central nervous system. Neuroscience 30:767-777.

Roltsch Hellard E, Binette A, Zhuang X, Lu J, Ma T, Jones B, Williams E, Jayavelu S, Wang J (2019) Optogenetic control of alcohol-seeking behavior via the dorsomedial striatal circuit. Neuropharmacology 155:89-97.

Shan Q, Ge M, Christie MJ, Balleine BW (2014) The acquisition of goal-directed actions generates opposing plasticity in direct and indirect pathways in dorsomedial striatum. J Neurosci 34:91969201.

Shiflett MW, Brown RA, Balleine BW (2010) Acquisition and performance of goal-directed instrumental actions depends on ERK signaling in distinct regions of dorsal striatum in rats. J Neurosci 30:2951-2959.

Smith JB, Klug JR, Ross DL, Howard CD, Hollon NG, Ko VI, Hoffman H, Callaway EM, Gerfen CR, Jin X (2016) Genetic-based dissection unveils the inputs and outputs of striatal patch and matrix compartments. Neuron 91:1069-1084.

Smith Y, Surmeier DJ, Redgrave P, Kimura M (2011) Thalamic contributions to basal ganglia-related behavioral switching and reinforcement. J Neurosci 31:16102-16106.

Stalnaker TA, Calhoon GG, Ogawa M, Roesch MR, Schoenbaum G (2010) Neural correlates of stimulus-response and response-outcome associations in dorsolateral versus dorsomedial striatum. Front Integr Neurosci 4:12.

Surmeier DJ, Ding J, Day M, Wang Z, Shen W (2007) D1 and D2 dopamine-receptor modulation of striatal glutamatergic signaling in striatal medium spiny neurons. Trends in Neurosciences 30:228235.

Volkow ND, Wang GJ, Telang F, Fowler JS, Logan J, Childress AR, Jayne M, Ma Y, Wong C (2006) Cocaine cues and dopamine in dorsal striatum: mechanism of craving in cocaine addiction. $J$ Neurosci 26:6583-6588.

Wall NR, De La Parra M, Callaway EM, Kreitzer AC (2013) Differential innervation of direct- and indirect-pathway striatal projection neurons. Neuron 79:347-360.

Wang H, Pickel VM (2002) Dopamine D2 receptors are present in prefrontal cortical afferents and their targets in patches of the rat caudate-putamen nucleus. J Comp Neurol 442:392-404.

Wang J, Lanfranco MF, Gibb SL, Yowell QV, Carnicella S, Ron D (2010) Long-lasting adaptations of the NR2B-containing NMDA receptors in the dorsomedial striatum play a crucial role in alcohol consumption and relapse. J Neurosci 30:10187-10198.

Wang J, Lanfranco MF, Gibb SL, Ron D (2011) Ethanol-mediated long-lasting adaptations of the NR2B-containing NMDA receptors in the dorsomedial striatum. Channels (Austin) 5:205-209.

Wang J, Cheng Y, Wang X, Roltsch Hellard E, Ma T, Gil H, Ben Hamida S, Ron D (2015) Alcohol elicits functional and structural plasticity selectively in dopamine D1 receptor-expressing neurons of the dorsomedial striatum. J Neurosci 35:11634-11643.

Wang L, Rangarajan KV, Gerfen CR, Krauzlis RJ (2018) Activation of striatal neurons causes a perceptual decision bias during visual change detection in mice. Neuron 97:1369-1381.e5.

Wang W, Dever D, Lowe J, Storey GP, Bhansali A, Eck EK, Nitulescu I, Weimer J, Bamford NS (2012) Regulation of prefrontal excitatory 
neurotransmission by dopamine in the nucleus accumbens core. $\mathrm{J}$ Physiol 590:3743-3769.

Wang Y, Goldman-Rakic PS (2004) D2 receptor regulation of synaptic burst firing in prefrontal cortical pyramidal neurons. Proc Natl Acad Sci USA 101:5093-5098.

Wei X, Ma T, Cheng Y, Huang CCY, Wang X, Lu J, Wang J (2018) Dopamine D1 or D2 receptor-expressing neurons in the central nervous system. Addict Biol 23:569-584.
Yin HH, Knowlton BJ (2004) Contributions of striatal subregions to place and response learning. Learn Mem 11:459-463.

Yin $\mathrm{HH}$, Knowlton BJ, Balleine BW (2005a) Blockade of NMDA receptors in the dorsomedial striatum prevents action-outcome learning in instrumental conditioning. Eur J Neurosci 22:505-512.

Yin HH, Ostlund SB, Knowlton BJ, Balleine BW (2005b) The role of the dorsomedial striatum in instrumental conditioning. Eur $\mathrm{J}$ Neurosci 22:513-523. 